\title{
On the linear stiffness of tension leg platforms
}

\author{
I. Senjanović, N. Hadžić, M. Tomić \\ University of Zagreb, Zagreb, Croatia
}

ABSTRACT: The tension leg platform (TLP) is a type of compliant offshore structures generally used for deep water oil exploration. The platforms are moored by very flexible tendons so that surge amplitude can achieve a large value. The platform hull is considered as a rigid body with six DOF. The total restoring stiffness plays a very important role in the TLP dynamic behavior. In present literature inadequate formulations of the stiffness have been used. The problem is to define realistic centre of rotations. The purpose of this paper is to present a consistent formulation of the stiffness matrix, derived from the general solution established for hydroelastic analysis of ship structures, as a specific case. In reality the total TLP stiffness in dynamic analysis consists of hydrostatic hull stiffness (pressure and gravity), and conventional and geometric tendon stiffness. The new stiffness is compared to the known ones, and discrepancies are analyzed and discussed.

\section{INTRODUCTION}

A tension leg platform (TLP) is a semi-submersible platform, moored by vertical pretensioned tendons or tethers (Baltrop 1998). The platform constitutive parts are pontoon, columns and deck with drilling equipment (Det norske Veritas 2005). Heave, roll and pitch have high natural frequencies due to high tendon axial stiffness. Surge, sway and yaw are compliant modes due to quite low tendon geometric stiffness. Vertical motions are excited by the first order wave forces, while horizontal motions appear due to the second order wave forces with very low frequency (Natvig \& Teigen 1993).

Stiffness plays very important role in dynamic analysis of TLPs (Adrezin et al. 1996). Platform can be considered as a rigid body with tendons as massless quasi-static springs. The hydrodynamic coefficients can be determined by Morison's equation or the radiation-diffraction theory, depending on the ratio of diameters of platform cylindrical segments and the wave length.

Since even linear stiffness is not formulated in the relevant literature in a consistent way, the new formulation is presented in Section 2, and its comparison with the known formulations is elaborated in Section 3. Additional comparison is done via numerical example, Section 4.

\section{LINEAR STIFFNESS}

\subsection{Definition of total stiffness}

Let us consider a double symmetric TLP with $N$ tendons, Figure 1. The origin of the coordinate system is located in the middle of the waterplane, and the motion components are shown in Figure 2. The platform is treated as a rigid body due to very high stiffness compared to that of tendons. The total stiffness consists of three parts:

$[K]_{P}=[K]^{C}+[C]+[K]^{G}$,

where $[K]^{C}$ is the conventional tendon stiffness, $[C]$ is the platform restoring stiffness with influence of tendons included, and $[K]^{G}$ is the tendon geometric stiffness.

\subsection{Conventional stiffness}

The tendons are steel pipes with negligibly small bending stiffness. Heave changes the tension of tendons so that the stiffness is the relation between vertical force and displacement:

$k_{33}^{C}=\frac{E A}{L}, \quad A=\sum_{n=1}^{N} A_{n}$.

The tension of tendons is also changed by roll and the corresponding stiffness is found from the moment as function of the roll angle. Since

$M_{x}=\sum_{n=1}^{N} F_{z}^{n} y_{n}, F_{z}^{n}=\frac{E A_{n}}{L} \delta_{z}^{n}$, and $\delta_{z}^{n}=y_{n} \varphi_{x}$,

one gets: 

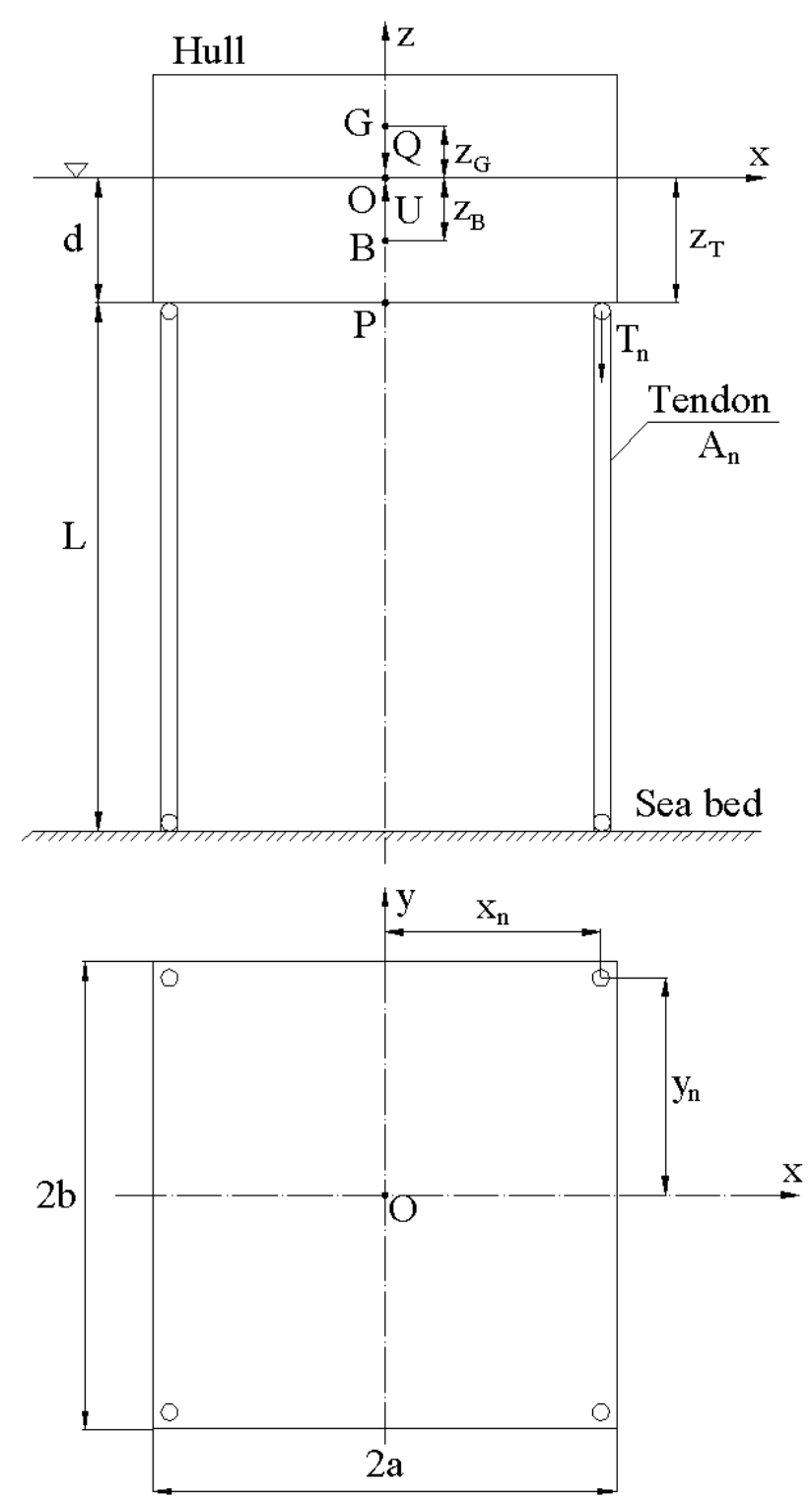

Figure 1. Double symmetric TLP.

$k_{44}^{C}=\frac{E I_{x}}{L}, \quad I_{X}=\sum_{n=1}^{N} A_{n} y_{n}^{2}$.

In the similar way, the pitch stiffness is:

$k_{55}^{C}=\frac{E I_{y}}{L}, \quad I_{y}=\sum_{n=1}^{N} A_{n} x_{n}^{2}$,

where $A, I_{x}$, and $I_{y}$ are the total cross-section area and moments of inertia about $\mathrm{x}$ and $\mathrm{y}$ axis of all tendons, respectively. Hence, set of tendons is considered as a beam with distinct fibers.

\subsection{Restoring stiffness}

Hydroelastic analysis of a deformable floating body is usually performed by the modal superposition method. The ordinary restoring stiffness consists of variation of hydrostatic pressure, variation of normal vector and natural mode and gravity part respectively, (Senjanović et al. 2009):

$$
\begin{aligned}
& C_{i j}^{o}=C_{i j}^{p}+C_{i j}^{n h}+C_{i j}^{m}, \\
& C_{i j}^{p}=\rho g \iint_{S} h_{k}^{i} h_{3}^{j} n_{k} \mathrm{~d} S, \\
& C_{i j}^{n h}=\rho g \iint_{S} Z h_{k}^{i} h_{l, l}^{j} n_{k} \mathrm{~d} S, \\
& C_{i j}^{m}=g \iiint_{V} \rho_{S} h_{k}^{i} h_{3, k}^{j} \mathrm{~d} S,
\end{aligned}
$$

where, according to the index notation, $h_{k, l}{ }^{i}$ is the $l$-th derivative of the $k$-th component of the natural mode $h^{i}, S$ is the wetted surface, $n_{k}$ are components of its normal vector, $V$ is the structure volume, while $\rho$ and $\rho_{s}$ are the water and structure density, respectively.

For the TLP platform only rigid body natural modes are of interest, and the general restoring stiffness is reduced to the formulas of ship hydrostatics for the floating and stability conditions, (SNAME 1988). In the case of double symmetric platform, the centroid of waterplane is located at the vertical line of the buoyancy and gravity centre and the restoring stiffness matrix is diagonal with the following heave, roll and pitch coefficients:

$$
\begin{aligned}
& C_{33}^{0}=\rho g A_{W L}^{0}, \\
& C_{44}^{0}=\rho g\left[I_{W L X}^{0}+V^{0}\left(z_{B}^{0}-z_{G}^{0}\right)\right], \\
& C_{55}^{0}=\rho g\left[I_{W L Y}^{0}+V^{0}\left(z_{B}^{0}-z_{G}^{0}\right)\right],
\end{aligned}
$$

where, $A_{W L}{ }^{0}, I_{W L X}{ }^{0}$ and $I_{W L Y}{ }^{0}$ are the waterplane area and its moments of inertia about $x$ and $y$ axis, respectively, $V^{0}$ is the buoyancy volume while $z_{B}{ }^{0}$ and $z_{G}{ }^{0}$ are coordinates of buoyancy and gravity centre, respectively.

The buoyancy, $U$, is larger than the platform weight, $Q$, due to tendon pretension forces, $T_{n}$, which, only for this purpose, can be treated as virtual lumped weights, Figure 3:

$\sum_{n=1}^{N} T_{n}=U-Q$.

The stiffness coefficients, Eqs. (10), (11) and (12) take the following form:

$$
\begin{aligned}
& C_{33}=\rho g A_{W L}, \\
& C_{44}=\rho g I_{W L X}+U z_{B}-Q z_{G}-\sum_{n=1}^{N} T_{n} z_{T}, \\
& C_{55}=\rho g I_{W L Y}+U z_{B}-Q z_{G}-\sum_{n=1}^{N} T_{n} z_{T},
\end{aligned}
$$




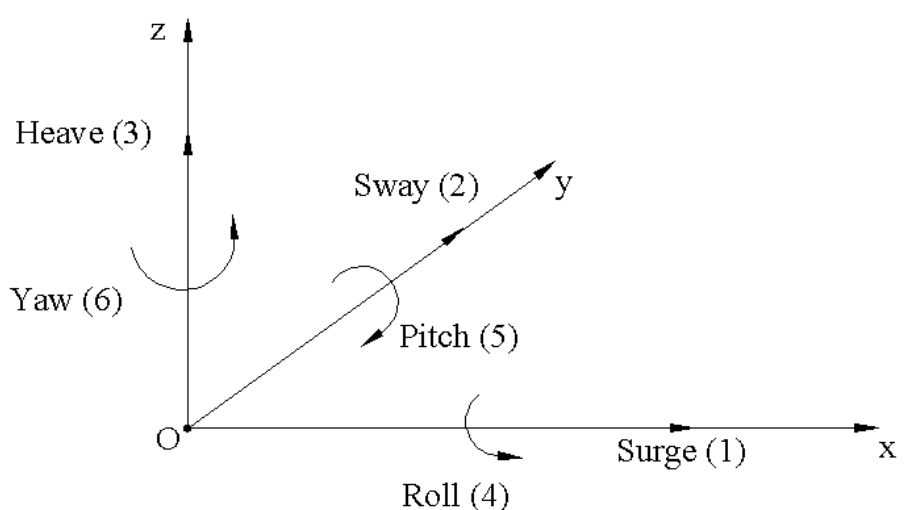

Figure 2. Motion components

where all quantities are related to the increased platform immersion due to the tendon forces.

\subsection{Geometric stiffness}

TLP can be translated horizontally in $x$ and $y$ direction and rotated about vertical $z$ axis. External forces are equilibrated with internal forces which depend on the tendon geometric stiffness. The stiffness can be determined by the general formulation of geometric stiffness written in the index notation (Huang \& Riggs 2000; Senjanović et al. 2010):

$k_{i j}^{G}=\iiint_{V} \sigma_{k l} h_{m, k}^{i} h_{m, l}^{j} \mathrm{~d} V$,

where $\sigma_{k l}$ is the stress tensor. In the considered case

$\sigma_{z z}=\sum_{n=1}^{N} \frac{T_{n}}{A_{n}}, \quad \mathrm{~d} V=\sum_{n=1}^{N} A_{n} \mathrm{~d} z$

and the surge mode

$$
h_{x}^{1}=\frac{(L+d+z)}{L}
$$

is defined in domain $-(L+d) \leq z \leq-d$, Figure 4a. Thus, one finds $h_{x, z}{ }^{1}=1 / L$ and further

$K_{11}^{G}=\sum_{n=1}^{N} \frac{T_{n}}{L}=K_{22}^{G}$.

The yaw mode of the $n$-th tendon, according to Figure 4b, is:

$h_{r}^{6}(n)=\frac{(L+d+z) r_{n}}{L}$

with components

$h_{x}^{6}(n)=\frac{(L+d+z) y_{n}}{L}$

$h_{y}^{6}(n)=\frac{(L+d+z) x_{n}}{L}$.
$K_{66}^{G}=\sum_{n=1}^{N} \frac{T_{n}\left(x_{n}^{2}+y_{n}^{2}\right)}{L}$.

\subsection{Total stiffness and mass matrix}

By summing up the terms of conventional, restoring and geometric stiffness, determined in previous sections, elements of the total stiffness matrix $[\mathrm{K}]_{P}$, Eq. (4), are obtained:

$$
\begin{aligned}
& K_{11}=K_{22}=\sum_{n=1}^{N} \frac{T_{n}}{L}, \quad K_{33}=\rho g A_{W L}+\frac{E A}{L}, \\
& K_{44}=\rho g I_{W L X}+U z_{B}-Q z_{G}-\sum_{n=1}^{N} T_{n} z_{T}+\frac{E I_{x}}{L}, \\
& K_{55}=\rho g I_{W L Y}+U z_{B}-Q z_{G}-\sum_{n=1}^{N} T_{n} z_{T}+\frac{E I_{y}}{L}, \\
& K_{66}=\sum_{n=1}^{N} \frac{T_{n}}{L}\left(x_{n}^{2}+y_{n}^{2}\right) .
\end{aligned}
$$

The total stiffness matrix is a diagonal one because the middle point of hull bottom is used as pole $P$, Figure 4 , for the platform rotations. As a result, there is no coupling between degrees of freedom through the stiffness.

On the other side, the mass matrix has some offdiagonal elements since the following inertia forces (designated with $i$ ) depend on both displacements and rotations:

$$
\begin{aligned}
& F_{x}^{i}=m \ddot{\delta}_{x}+m\left(z_{G}-z_{T}\right) \ddot{\varphi}_{y} \\
& F_{y}^{i}=m \ddot{\delta}_{y}+m\left(z_{G}-z_{T}\right) \ddot{\varphi}_{x} \\
& M_{x}^{i}=m\left(z_{G}-z_{T}\right) \ddot{\delta}_{y}+J_{x}^{P} \ddot{\varphi}_{x} \\
& M_{y}^{i}=m\left(z_{G}-z_{T}\right) \ddot{\delta}_{x}+J_{y}^{P} \ddot{\varphi}_{y}
\end{aligned}
$$

where

$J_{x}^{P}=J_{x}^{G}+m\left(z_{G}-z_{T}\right)^{2}$

$J_{y}^{P}=J_{y}^{G}+m\left(z_{G}-z_{T}\right)^{2}$

are the mass moments of inertia. The mass matrix with respect to the pole $P$, reads:

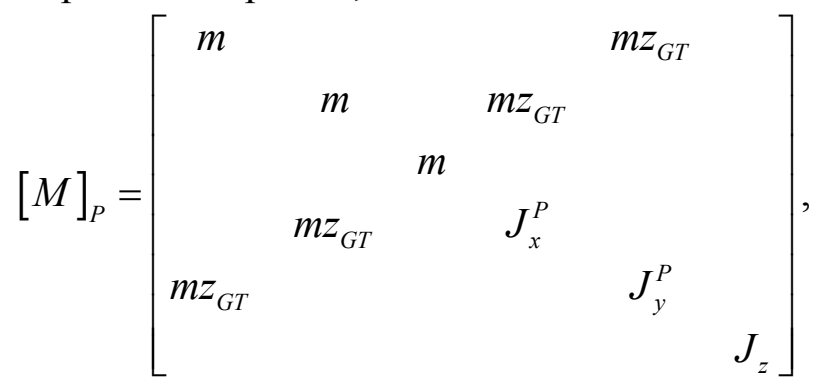

In this case, Eq. (16) gives 


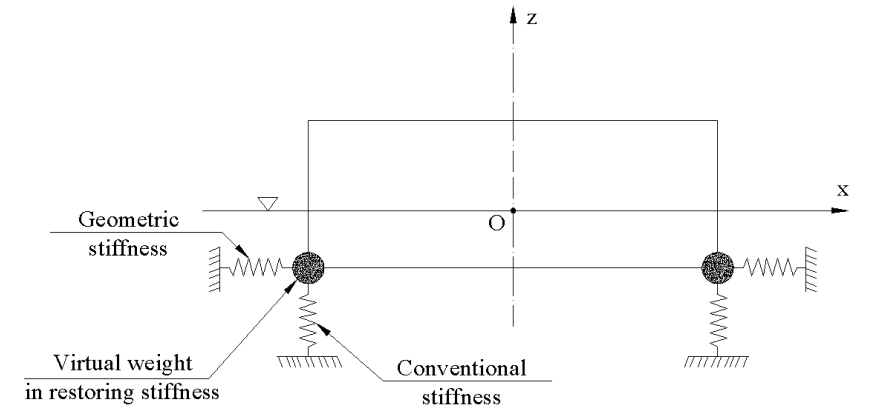

Figure 3. Stiffness model of TLP

where $z_{G T}=z_{G}-z_{T}$. Due to the off-diagonal terms of matrix $[\mathrm{M}]_{P}$, the vibrations are coupled through the mass matrix.

If the center of gravity $G$ is used as the reference point, the following relations between displacements exist:

$\delta_{x}^{P}=\delta_{x}^{G}-\left(z_{G}-z_{T}\right) \varphi_{y}, \quad \delta_{y}^{P}=\delta_{y}^{G}-\left(z_{G}-z_{T}\right) \varphi_{x}$.

Hence, the displacement transformation matrix reads:

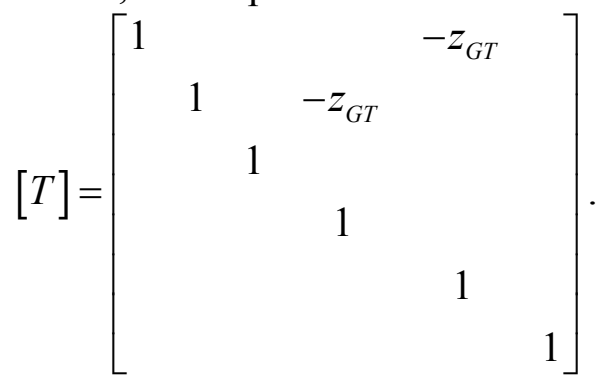

Now, the stiffness and mass matrix can be adapted to the new coordinate system in the way well known in the finite element method, which is based on the fact that the total energy of a structure does not depend on the chosen coordinate system, (Zienkiewicz 1971):

$[K]_{G}=[T]^{T}[K]_{P}[T], \quad[M]_{G}=[T]^{T}[M]_{P}[T]$.

$(38 \mathrm{a}, \mathrm{b})$

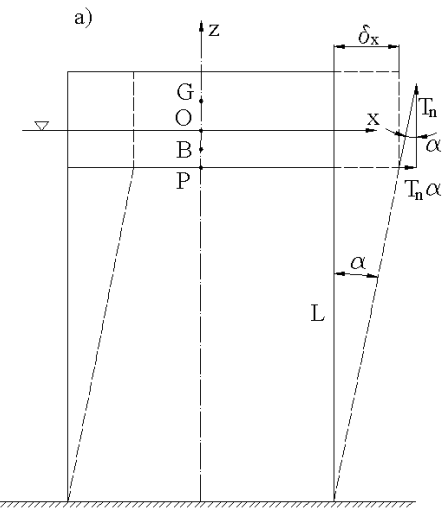

b) Platform hottom

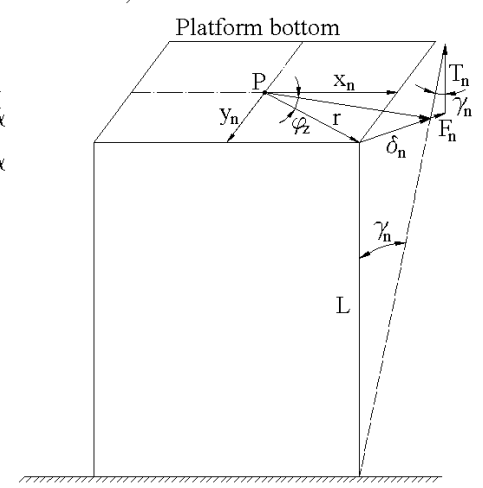

Figure 4. Surge and sway of TLP
Thus, one finds:

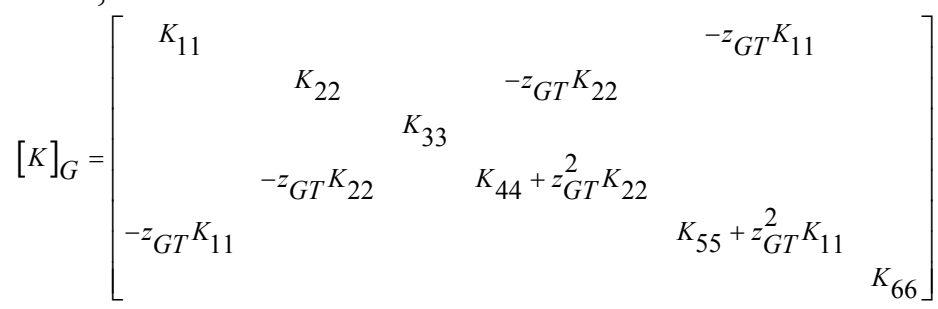

while the mass matrix becomes diagonal:

$$
[M]_{G}=\left[\begin{array}{ccccc}
m & & & & \\
& m & & & \\
& m & & & \\
& & J_{x}^{G} & & \\
& & & J_{y}^{G} & \\
& & & & J_{z}
\end{array}\right] \text {. }
$$

In direct analysis of ship motion in seaway, it is assumed that vessel rotates about the centroid of the waterplane. If the same assumption is accepted for TLP, the stiffness and mass matrix can be transformed in the same manner and both have off-diagonal elements.

\section{COMPARISON OF THE KNOWN STIFFNESS MATRICES WITH THE NEW ONE}

\subsection{Stiffness from Malenica, 2003}

The linear stiffness matrix is derived for the tendon top points and arbitrary origin of the coordinate system.

$[C]=\left[C_{W L}\right]+\sum_{n=1}^{N}\left[C^{P_{0}^{n}}\right]+\sum_{n=1}^{N}\left[C^{P_{1}^{n}}\right]$,

where

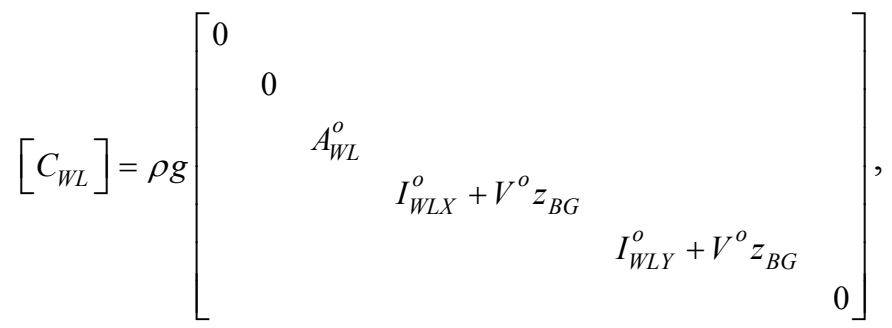

and

$$
\left[C^{P_{0}^{n}}\right]=\left[\begin{array}{cccccc}
0 & & & & & \\
& 0 & & & & \\
& & 0 & & & \\
& & -T_{n} z_{G}^{n} & & \\
& & & -T_{n} z_{G}^{n} & \\
& & & & 0
\end{array}\right]
$$




$$
\begin{aligned}
& {\left[C^{P_{1}^{n}}\right]=\left[\begin{array}{cc}
{\left[k^{n}\right]} & -\left[k^{n}\right]\left[V^{n}\right] \\
{\left[V^{n}\right]\left[k^{n}\right]} & -\left[V^{n}\right]\left[k^{n}\right]\left[V^{n}\right]
\end{array}\right],} \\
& {\left[k^{n}\right]=\left[\begin{array}{ccc}
k_{11}^{n} & \\
& k_{22}^{n} & \\
&
\end{array}\right],} \\
& {\left[V^{n}\right]=\left[\begin{array}{ccc}
0 & -z_{G}^{n} & y_{G}^{n} \\
z_{G}^{n} & 0 & -x_{G}^{n} \\
-y_{G}^{n} & x_{G}^{n} & 0
\end{array}\right],} \\
& {\left[k^{n}\right]\left[V^{n}\right]=\left[\begin{array}{ccc}
0 & -z_{G}^{n} k_{11}^{n} & y_{G}^{n} k_{11}^{n} \\
z_{G}^{n} k_{22}^{n} & 0 & -x_{G}^{n} k_{22}^{n} \\
-y_{G}^{n} k_{33}^{n} & x_{G}^{n} k_{33}^{n} & 0
\end{array}\right],}
\end{aligned}
$$

$\left[V^{n}\right]\left[k^{n}\right]\left[V^{n}\right]=$

$$
\left[\begin{array}{ccc}
-\left(z_{G}^{n}\right)^{2} k_{22}^{n}-\left(y_{G}^{n}\right)^{2} k_{33}^{n} & x_{G}^{n} y_{G}^{n} k_{33}^{n} & -x_{G}^{n} z_{G}^{n} k_{22}^{n} \\
-x_{G}^{n} y_{G}^{n} k_{33}^{n} & -\left(z_{G}^{n}\right)^{2} k_{11}^{n}-\left(x_{G}^{n}\right)^{2} k_{33}^{n} & y_{G}^{n} z_{G}^{n} k_{11}^{n} \\
x_{G}^{n} z_{G}^{n} k_{22}^{n} & y_{G}^{n} z_{G}^{n} k_{11}^{n} & -\left(y_{G}^{n}\right)^{2} k_{11}^{n}-\left(x_{G}^{n}\right)^{2} k_{22}^{n}
\end{array}\right] \text {, }
$$

$k_{11}^{n}=k_{22}^{n}=\frac{T_{n}}{L}, k_{33}^{n}=\frac{E A_{n}}{L}$,

$x_{G}^{n}=x^{n}-x_{G}, y_{G}^{n}=y^{n}-y_{G}, z_{G}^{n}=z^{n}-z_{G}$.

In the case of a double symmetric platform $x_{G}{ }^{n}= \pm a$, $y_{G}{ }^{n}= \pm b, \quad z_{G}{ }^{n}=z_{T G}$, Figure 1, and all off-diagonal elements in the resulting matrix, Eq. (41), vanish. By taking into account the relations

$$
\begin{aligned}
& \rho g V^{0}=Q, \quad U=\sum_{n=1}^{N} T_{n}+Q, \\
& z_{B G}=z_{B}-z_{G}, \quad z_{T G}=z_{T}-z_{G}, \\
& A b^{2}=I_{X}, \quad A a^{2}=I_{Y}
\end{aligned}
$$

one can write for diagonal elements

$$
\begin{aligned}
& C_{11}=C_{22}=\sum_{n=1}^{N} \frac{T_{n}}{L}, C_{33}=\rho g A_{W L}^{o}+\frac{E A}{L}, \\
& C_{44}=\rho g I_{W L X}^{o}+U z_{B}-Q z_{G}-\sum_{n=1}^{N} T_{n} z_{T}-\sum_{n=1}^{N} T_{n} z_{B G}+\sum_{n=1}^{N} \frac{T_{n}}{L} z_{T G}^{2}+\frac{E I_{X}}{L},
\end{aligned}
$$

$C_{55}=\rho g I_{W L Y}^{o}+U z_{B}-Q z_{G}-\sum_{n=1}^{N} T_{n} z_{T}-\sum_{n=1}^{N} T_{n} z_{B G}+\sum_{n=1}^{N} \frac{T_{n}}{L} z_{T G}^{2}+\frac{E I_{Y}}{L}$,

$C_{66}^{P}=\sum_{n=1}^{N} \frac{T_{n}}{L}\left(a^{2}+b^{2}\right)$.

By comparing Eqs. (52) - (55) with Eqs. (25) - (28) some differences can be noticed. The $5^{\text {th }}$ and $6^{\text {th }}$ terms in Eqs. (53) and (54) are additional.

\subsection{Stiffness from Jain, 1997}

Nonlinear stiffness matrix presented in (Jain 1997) is ordinary used for dynamic analysis of TLP. It is specified with respect to the centre of gravity:

$$
[K]_{G}^{E}=\left[\begin{array}{llllll}
K_{11} & & & & & \\
& K_{22} & & & & \\
K_{31} & K_{32} & K_{33} & K_{34} & K_{35} & K_{36} \\
& K_{42} & & K_{44} & & \\
K_{51} & & & & K_{55} & \\
& & & & & K_{66}
\end{array}\right] .
$$

The linear part of Eq. (56) reads

$$
[K]_{G}^{L}=\left[\begin{array}{cccccc}
K_{11}^{L} & & & & & \\
& K_{22}^{L} & & & & \\
& & K_{33}^{L} & & & \\
& K_{42}^{L} & & K_{44}^{L} & & \\
K_{51}^{L} & & & & K_{55}^{L} & \\
& & & & & K_{66}^{L}
\end{array}\right] \text {, }
$$

where elements $K_{11}{ }^{L}, K_{22}{ }^{L}, K_{33}{ }^{L}$ and $K_{66}{ }^{L}$ are equal to Eqs. (25a, b) and (28), while the remaining elements are

$$
\begin{aligned}
& K_{44}^{L}=\rho g A_{W L}+U z_{G}-Q z_{G}-\sum_{n=1}^{N} T_{n} z_{T}+\frac{E I_{X}}{L}, \\
& K_{55}^{L}=\rho g A_{W L}+U z_{G}-Q z_{G}-\sum_{n=1}^{N} T_{n} z_{T}+\frac{E I_{Y}}{L} .
\end{aligned}
$$

By comparing Eqs. (58) and (59) with Eqs. (26) and (27) it is noticed that the coordinate of the gravity center $z_{G}$ is accompanied to the buoyancy $U$ instead of its own $z_{B}$. Since Eq. (57) is derived with respect to the gravity center it should have additional terms $K_{15}{ }^{L}$ and $K_{24}{ }^{L}$ like Eq. (39).

\subsection{Stiffness from Low, 2009}

Recently, a new formulation of nonlinear stiffness matrix, based on energy approach, is presented in (Low 2009). Its linear part reads

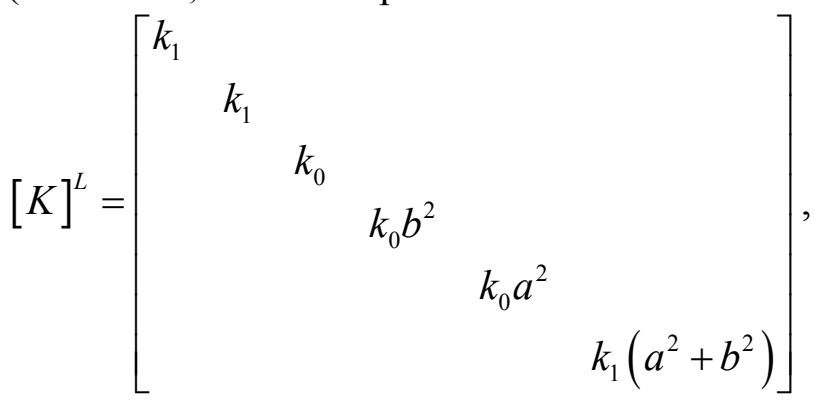

where 
$k_{0}=\frac{E A}{L}, k_{1}=\sum_{n=1}^{N} \frac{T_{n}}{L}$.

It is obvious that only the tendon contribution is taken into account, while the restoring stiffness is ignored.

\section{NUMERICAL EXAMPLE}

Outlined theory is illustrated by analyzing a TLP spar floater without the installed wind turbine (Withee 2004). Hydrodynamic part of fluid loading (added mass, radiation damping, Froude-Krylov and diffraction loads) and the equations of motion (solved for the centre of gravity of the floater) are calculated using the Bureau Veritas HYDROSTAR software (Chen 2004).

Table 1. Particulars of a TLP spar floater

\begin{tabular}{|l|r|l|r|}
\hline Spar diameter & $4 \mathrm{~m}$ & Water depth & $200 \mathrm{~m}$ \\
Spar draft & $10 \mathrm{~m}$ & Mass & $49002.3 \mathrm{~kg}$ \\
Thickness & $25.3 \mathrm{~mm}$ & Buoyant mass & $149887.8 \mathrm{~kg}$ \\
Spoke length & $5 \mathrm{~m}$ & COG & $-6.698 \mathrm{~m}$ \\
Spoke width & $1 \mathrm{~m}$ & COB & $-5.687 \mathrm{~m}$ \\
Line diameter & $50 \mathrm{~mm}$ & Jxx & $1140080.9 \mathrm{~kg} \mathrm{~m}^{\wedge} 2$ \\
Number of lines & $1 \mathrm{per}$ spoke & Jyy & $1140080.9 \mathrm{~kg} \mathrm{~m}^{\wedge} 2$ \\
Line length & $190 \mathrm{~m}$ & Jzz & $451478 \mathrm{~kg} \mathrm{~m}^{\wedge} 2$ \\
\hline
\end{tabular}

The mean wetted surface of a TLP spar floater is discretized into 2272 panels. Two distinctive cases were analyzed, one using high modulus polyester tendons (HMPE, $E=2.510^{10} \mathrm{~N} / \mathrm{m}^{2}$ ) and the other using the usual steel tendons (STEEL, E=2.06 $10^{11} \mathrm{~N} / \mathrm{m}^{2}$ ). HMPE tendons are used in order to lower the stiffness and thus allow larger oscillation amplitudes. Frequency domain responses (1st order motion transfer functions) were calculated for a range of frequencies in $1 \mathrm{~m}$ head waves (180 deg wave heading). Please note that the ratio between the response amplitude and wave amplitude is called transfer function in this paper.

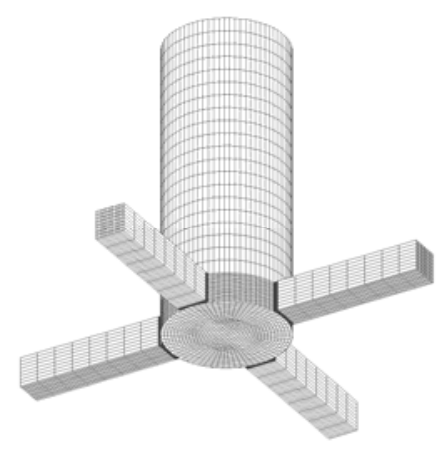

Figure 5. Panel model of TLP

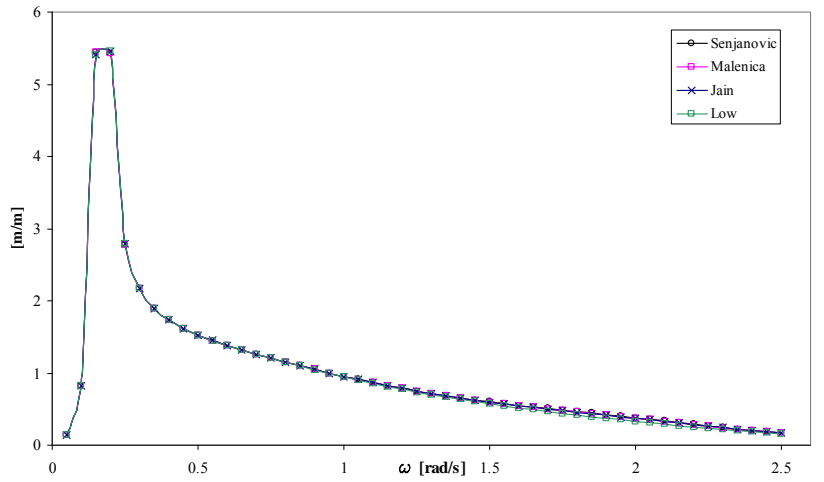

Figure 6. TLP surge transfer function (HMPE tendons)

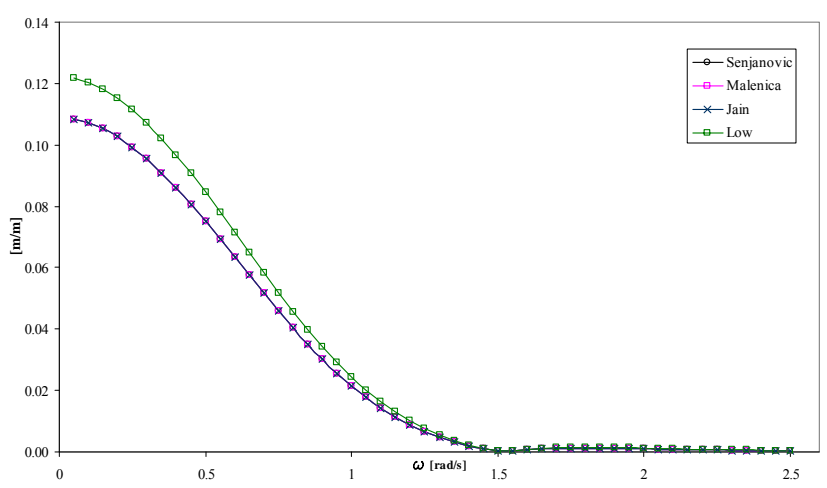

Figure 7. TLP heave transfer function (HMPE tendons)

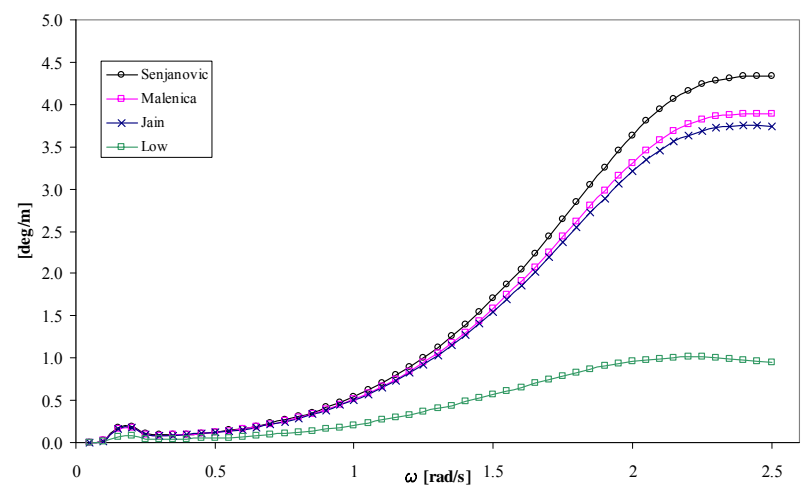

Figure 8. TLP pitch transfer function (HMPE tendons)

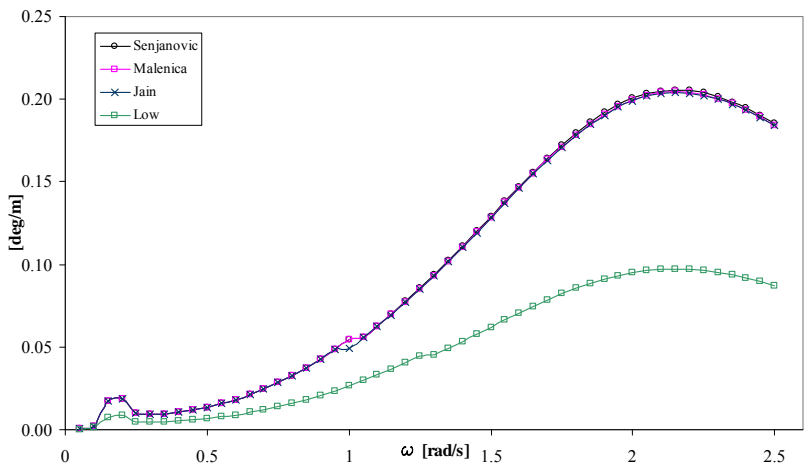

Figure 9. TLP pitch transfer function (STEEL tendons)

In Figure 6 one can see that there are almost no differences in the surge due to different stiffness formulations and obviously these is the most important mode for TLP's. Low's stiffness formulation results with slightly larger values for the 
heave response, Figure 7, than the other ones that are again very close to each other. The largest differences are obtained for the rotational modes of motion as can be noted from the Figure 8. That particular case corresponds to HMPE tendons and the lower elasticity obviously will pronounce differences (as compared to steel tendons in Figure 9 were the different formulations give practically identical results). One should also note that the coupling between the modes of motion is influenced by the distance between the center of gravity and the center of buoyancy, so one would expect slightly larger differences between the stiffness formulations by Senjanovic and Malenica for the rotational modes, if the wind turbine itself was included in the model.

\section{CONCLUSION}

In this paper, the consistent linear stiffness for dynamic analysis of TLP's is derived in a systematic and physically transparent way. It comprises platform restoring stiffness, tendon conventional stiffness and tendon geometric stiffness. The first two components are important for the vertical motions, while the third one is related to the horizontal motions. Comparison of the known stiffness matrices with the new one shows some differences of the former. Their influence on response will be analyzed in further investigation by numerical examples. In general there is a good agreement between different formulations, especially if steel tendons are used. Therefore, in analyzing TLP's one should use formulation which appears to be physically consistent.

\section{REFERENCES}

Adrezin, R. et al. 1996. Dynamic response of compliant offshore structures - review, J. Aerosp. Eng., 9(4), pp. 114 - 131.

Baltrop, N.D.P. 1998. Floating structures: a guide for design and analysis, Vols. 1 and 2, Oilfield Publications Ltd.

Chen, X. 2004. Hydrodynamics in offshore and naval applications - part I., Keynote Lecture, 6th International Conference on Hydrodynamics, Perth, Australia

Det norske Veritas, 2005. DNV - OS - C105 Structural design of TLP (LRFD method).

Huang, L.L. \& Riggs, H.R. 2000. The hydrostatic stiffness of flexible floating structures for linear hydroelasticity, Marine Structures, 13, pp. 91-106.

Jain, A.K. 1997. Nonlinear coupled response of offshore tension leg platform to regular wave forces, Ocean Engineering, 24(7), pp. 577-593.
Low, M.Y. 2009. Frequency domain analysis of a tension leg platform with statistical linearization of the tendon restoring forces, Marine Structures, 22, pp. 480-503.

Malenica, Š. 2003. Some aspects of hydrostatic calculations in linear seakeeping, International Conference on Ship and Shipping Research, NAV, Palermo, Italy.

Natvig, B.J. \& Teigen, P. 1993. Review of hydrodynamic challenges in TLP Design, Proceedings of the $3^{\text {rd }}$ International Offshore and Polar Engineering Conference, Singapure, pp. $294-302$.

Senjanović, I. et al. 2009. Ship hydroelastic analysis with sophisticated beam model and consistent restoring stiffness, Proceedings of Hydroelasticity in Marine Technology, Southampton.

Senjanović, I. et al. 2010. Some aspects of geometric stiffness modeling in the hydroelastic analysis of ship structures, Transactions of FAMENA, Vol. 34, No. 4.

SNAME, 1988. Principles of Naval Architecture, Jersey City, NJ, The Society of Naval Architects and Marine Engineers.

Zienkiewicz, O.C. 1971. The Finite Element Method in Engineering Science, London: McGraw - Hill.

Withee, J.E. 2004. Fully coupled dynamic analysis of a floating wind turbine system, $\mathrm{PhD}$ thesis, MIT, USA 